\title{
Ecologia da Paisagem de uma Bacia Hidrográfica dos Tabuleiros Costeiros do Brasil
}

\author{
Marcia Fernandes ${ }^{1}$, Milton Fernandes ${ }^{2}$, André Almeida ${ }^{3}$, \\ Maria Isidória da Silva Gonzaga ${ }^{4}$, Fábio Gonçalves ${ }^{5}$ \\ ${ }^{1}$ Departamento de Ciências Florestais e da Madeira - DCFM, Universidade Federal do Espírito Santo - UFES, \\ Jerônimo Monteiro/ES, Brasil \\ ${ }^{2}$ Departamento de Ciências Florestais - DCF, Universidade Federal de Sergipe - UFS, São Cristóvão/SE, Brasil \\ ${ }^{3}$ Departamento de Engenharia Agrícola - DEAGRI, Universidade Federal de Sergipe - UFS, São Cristóvão/SE, Brasil \\ ${ }^{4}$ Departamento de Engenharia Agronômica - DEA, Universidade Federal de Sergipe - UFS, São Cristóvão/SE, Brasil \\ ${ }^{5}$ Canopy Remote Sensing Solutions, Florianópolis/SC, Brasil
}

\section{RESUMO}

O objetivo deste trabalho foi quantificar o uso da terra e as métricas da paisagem da Bacia Hidrográfica do Rio Piauitinga, SE, a partir de imagens orbitais de alta resolução espacial. Foi utilizada uma imagem RapidEye com resolução espacial de 5 metros para mapear o uso e cobertura da terra. Foram consideradas as métricas de ecologia da paisagem ao nível de classe. Além disso, foram realizadas simulações da distância de borda nos fragmentos florestais remanescentes. A bacia apresenta uma paisagem dominada por pastagens $(50,43 \%)$. A vegetação nativa é a segunda maior classe de uso $(23,79 \%)$, porém está fragmentada, com numerosos fragmentos de tamanho pequeno e com grande efeito de borda. O aumento da distância de borda reduz a área central, principalmente dos fragmentos da classe muito pequeno, sendo indicado o estabelecimento de corredores ecológicos.

Palavras-chave: RapdiEye, sistemas de informações geográficas, Fragstats.

\section{Landscape Ecology of a Watershed in the Brazilian Coastal Flat Lands}

\begin{abstract}
This study aimed to quantify the land use and extract landscape metrics in the Piauitinga basin, $\mathrm{SE}$, using satellite images with high spatial resolution. We used a RapidEye image with spatial resolution of five meters to map the land use and land cover. The landscape ecology metrics were calculated at the class level. In addition, simulations were performed to understand the edge effect for the remaining forest fragments. The basin presents a landscape dominated by pasture land (50.43\%). Native vegetation represents the second largest class (23.79\%), but is fragmented, with numerous small fragments showing a large edge effect. The increase in the edge distance reduces the central area, especially for fragments of the smallest size class, which indicates the establishment of ecological corridors.
\end{abstract}

Keywords: RapidEye, geographic information systems, Fragstats. 


\section{INTRODUÇÃO}

O Bioma Mata Atlântica, que cobria originalmente grande parte do território brasileiro, encontra-se hoje altamente fragmentado, composto por remanescentes muito pequenos, isolados e com baixa diversidade de hábitats (Ribeiro et al., 2009). Segundo Metzger (2009), a Mata Atlântica é, provavelmente, uma das florestas mais fragmentadas, quando comparada com as demais florestas tropicais. Atualmente, cerca de $12 \%$ da sua área original permanecem em manchas pequenas e isoladas, sendo que $84 \%$ correspondem a fragmentos menores que 50 ha (Ribeiro et al., 2009). Em Sergipe, restam cerca de $8 \%$ da faixa original de Mata Atlântica que, de acordo com alguns estudos, são áreas que permanecem sob constante pressão antrópica (Ferreira et al., 2011).

A fragmentação dos remanescentes florestais e o uso da terra muitas vezes sem os cuidados necessários para mitigação dos impactos decorrentes das atividades antrópicas geram ecossistemas fragilizados por fenômenos como o efeito de borda, ou seja, alterações localizadas nas áreas de contato entre a matriz antropizada e o remanescente florestal (Etto et al., 2013).

As mudanças ocorridas em uma paisagem podem ser estudadas por meio da avaliação de índices ou métricas da paisagem para caracterização quantitativa, que permitem também comparar paisagens, identificar diferenças e determinar relações entre os processos funcionais e os padrões dessas paisagens (Cabacinha et al., 2010). A análise dos padrões da fragmentação florestal pode ser feita por meio das métricas da paisagem, consideradas como base para análises que visam quantificar a estrutura da paisagem, pois fornecem suporte científico para evidenciar quantidade e qualidade das manchas, com base na Ecologia da Paisagem, considerando principalmente o tamanho do fragmento, a área central, a forma e o grau de seu isolamento (Silva \& Souza, 2014).

Por meio de técnicas de Sistemas de Informação Geográfica (SIG) e Sensoriamento Remoto (SR) é possível calcular os índices espaciais utilizados em Ecologia da Paisagem, que são capazes de descrever o nível de uniformidade ou fragmentação da paisagem. Esse tipo de análise pode permitir a realização de estudos da paisagem relacionados à biodiversidade, os quais não seriam possíveis de serem realizados somente em campo (Souza et al., 2014).

Banks-Leite et al. (2011), em seus estudos do efeito da fragmentação da Mata Atlântica, obtiveram grande correlação dos impactos da modificação da paisagem na composição da comunidade, utilizando indicadores baseados na paisagem (métricas ou índices) para avaliar conservação das áreas de Mata Atlântica.

Diante do exposto, o presente trabalho teve como objetivo analisar e quantificar o uso da terra e as métricas da paisagem Bacia do Rio Piauitinga, SE, a partir de imagens orbitais com alta resolução espacial.

\section{MATERIAL E MÉTODOS}

\subsection{Localização e caracterização da área de estudo}

O trabalho foi realizado na Bacia Hidrográfica do Rio Piauitinga, região sul do Estado de Sergipe, entre as coordenadas geográficas $10^{\circ} 34^{\prime}$ e $10^{\circ} 45^{\prime} \mathrm{S}$ e $37^{\circ} 22^{\prime}$ e $37^{\circ} 34^{\prime} \mathrm{W}$ (Figura 1). A bacia ocupa área de aproximadamente $412,66 \mathrm{~km}^{2} \mathrm{e}$ a cobertura vegetal da região é típica do Bioma Mata Atlântica. O clima é do tipo Megatérmico Subúmido, com temperatura média anual de $28^{\circ} \mathrm{C}$, mínima de $22,3{ }^{\circ} \mathrm{C}$ e máxima de $26^{\circ} \mathrm{C}$. A média pluviométrica anual é de aproximadamente $1.300 \mathrm{~mm}$, com chuvas concentradas no período de maio a agosto. Os solos da bacia são predominantemente dos tipos Argissolo e Cambissolo (Magalhães et al., 2012).

\subsection{Uso e cobertura da terra}

O mapeamento de uso e cobertura da terra foi realizado a partir de imagens orbitais RapidEye (RE2 e RE4) com resolução espacial de 5 metros e nível de processamento $3 \mathrm{~A}$, com correção geométrica e radiométrica. Maiores informações: consultar manual técnico do produto em Planet Labs Inc. (2016). As imagens foram cedidas pelo Ministério do Meio Ambiente - MMA.

Foram utilizadas três diferentes cenas do ano de 2013 (dias 25 de janeiro e 7 e 12 de fevereiro), linhas $339 / 340$ e colunas $21 / 22$. A imagem possui como referência espacial o Datum WGS 1984 UTM Zona 24S, formato tiff, com profundidade de pixel de 16 bit, fonte contínua e cinco bandas espectrais: azul (440-510 nm); verde (520-590 nm); vermelho (630-685 nm); borda 


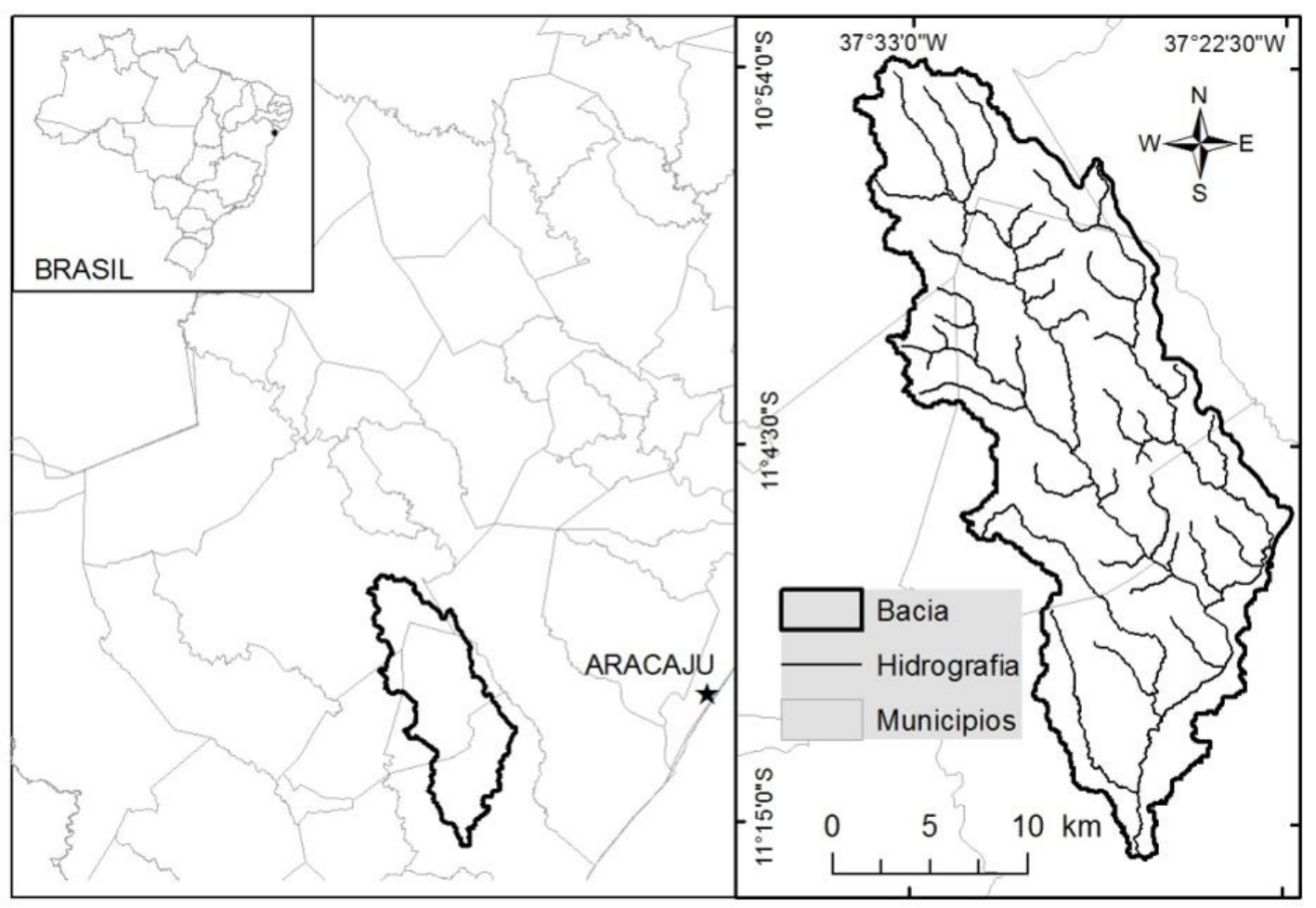

Figura 1. Localização da área de estudo.

Figure 1. Study area.

do vermelho (690-730 nm); e infravermelho próximo (760-850 nm).

O mapeamento de uso e cobertura da terra foi realizado de forma manual via tela do computador, a partir das composições das bandas azul, verde e vermelho e verde, vermelho e infravermelho próximo, na escala de 1:30.000. Foram utilizadas como elementos de interpretação visual das imagens tonalidades, formas e texturas. Foram definidas oito classes de uso da terra: área urbana, aquíferos superficiais, cultura agrícola, floresta plantada, nuvens, pastagem, solo exposto e vegetação nativa.

\subsection{Métricas da paisagem}

Para quantificar os índices espaciais e as métricas da paisagem foi utilizado o software Fragstats ${ }^{\circledR}$ versão 4.2 (McGarigal, 2013). A seleção dos índices espaciais foi baseada em preceitos ecológicos de acordo com Metzger (2009). Para o cálculo das métricas da paisagem (Tabela 1), foram selecionados índices ao nível de classe que possibilitaram quantificar os elementos da paisagem, estimando-se assim área, densidade, borda, área central, forma, proximidade e isolamento, contágio e dispersão (McGarigal, 2013).

Para melhor comparação entre o grau de conservação, o efeito de borda e o tamanho dos fragmentos florestais da classe vegetação nativa mapeados neste estudo foi realizada a análise dos índices de ecologia da paisagem para área central de cada uma das classes de tamanho: muito pequenos ( $<5 \mathrm{ha})$, pequenos (5-10 ha), médios (10-100 ha) e grandes (> $100 \mathrm{ha}$ ).

$\mathrm{Na}$ simulação do efeito de borda nas diferentes classes de tamanho dos fragmentos florestais foram utilizadas as seguintes distâncias de borda: 30 m, $60 \mathrm{~m}$ e $90 \mathrm{~m}$, pois acima de $100 \mathrm{~m}$ o fragmento encontra-se totalmente sob efeito de borda.

\section{RESULTADOS E DISCUSSÃO}

\subsection{Mapeamento de uso e cobertura da terra}

As oito classes de uso e cobertura da terra identificadas a partir da interpretação das imagens orbitais RapdiEye podem ser observadas na Tabela 1 
Tabela 1. Métricas espaciais utilizadas na quantificação da estrutura da paisagem na Bacia do Rio Piauitinga, SE. Table 1. Spatial metrics used to quantify the landscape structure in Piauitinga River basin-SE.

\begin{tabular}{|c|c|c|}
\hline Métrica & Sigla e intervalo (unidade) & Grupo \\
\hline Número de fragmentos & $\mathrm{NP} \geq 1$ (adimensional) & \multirow{7}{*}{$\begin{array}{c}\text { Área, densidade e } \\
\text { borda }\end{array}$} \\
\hline$\%$ de fragmentos de mesma classe & $0<$ PLAND $\leq 100(\%)$ & \\
\hline$\%$ da área do maior fragmento & $0<\mathrm{LPI} \leq 100(\%)$ & \\
\hline Área média dos fragmentos & AREA_MN >0 (ha) & \\
\hline Área de classe & $\mathrm{CA}>0$ (ha) & \\
\hline Total de bordas & $\mathrm{TE} \geq 0$ (ha) & \\
\hline Densidade de bordas & $\mathrm{ED} \geq 0\left(\mathrm{~m} \cdot \mathrm{ha}^{-1}\right)$ & \\
\hline Índice de forma médio & SHAPE_MN $\geq 1$ (adimensional) & Forma \\
\hline Total da área central (ha) & TCA $\geq 0$ (ha) & \multirow{3}{*}{ Área central } \\
\hline Número de áreas centrais disjuntas & NDCA $\geq 0$ (adimensional) & \\
\hline Índice de área central médio & $0<$ CAI_MN $\leq 100(\%)$ & \\
\hline Proximidade média entre classes & PROX_MN $\geq 0$ (adimensional) & \multirow{2}{*}{$\begin{array}{l}\text { Proximidade e } \\
\text { isolamento }\end{array}$} \\
\hline Distância média do vizinho mais próximo & ENN_MN $\geq 0(\mathrm{~m})$ & \\
\hline Conectividade & $0<$ COHESION $<100(\%)$ & $\begin{array}{l}\text { Contágio e } \\
\text { dispersão }\end{array}$ \\
\hline
\end{tabular}

e Figura 2. Os resultados demonstram que a imagem RapidEye possibilitou a identificação e diferenciação dos distintos usos existentes na Bacia Hidrográfica do rRo Piauitinga de modo satisfatório para os objetivos do estudo.

Área urbana, aquíferos superficiais e floresta plantada, com $1,87 \%, 0,07 \%$ e $0,98 \%$, respectivamente (Tabela 2), foram as menores classes de uso da terra mapeadas. Resultado semelhante também foi encontrado no trabalho realizado por Nascimento \& Abreu (2012) no município de Pedro Afonso, nordeste do Estado de Tocantins.

Considerando a matriz da paisagem da área de estudo, a classe cultura agrícola encontra-se concentrada na porção alta da bacia, região onde está localizada boa parte das nascentes, responsáveis pelo suprimento de água dos cursos d'água no período de estiagem (Figura 2). Dessa forma, torna-se necessário o reflorestamento da parte alta da Bacia do Rio Piauitinga e a adoção de práticas conservacionistas nas áreas agricultáveis. Segundo Magalhães et al. (2012), a situação das nascentes da Bacia do Rio Piauitinga é preocupante, pois $22 \%$ estão perturbadas e as demais degradadas (88\%).

Para as classes pastagem e vegetação nativa (Mata Atlântica), foram observadas as maiores ocorrências, com 50,43\% e 23,79\%, respectivamente. As áreas de pastagem ocupam metade da área total da bacia (Tabela 2 e Figura 2). O grande percentual de pastagem na bacia pode comprometer a capacidade

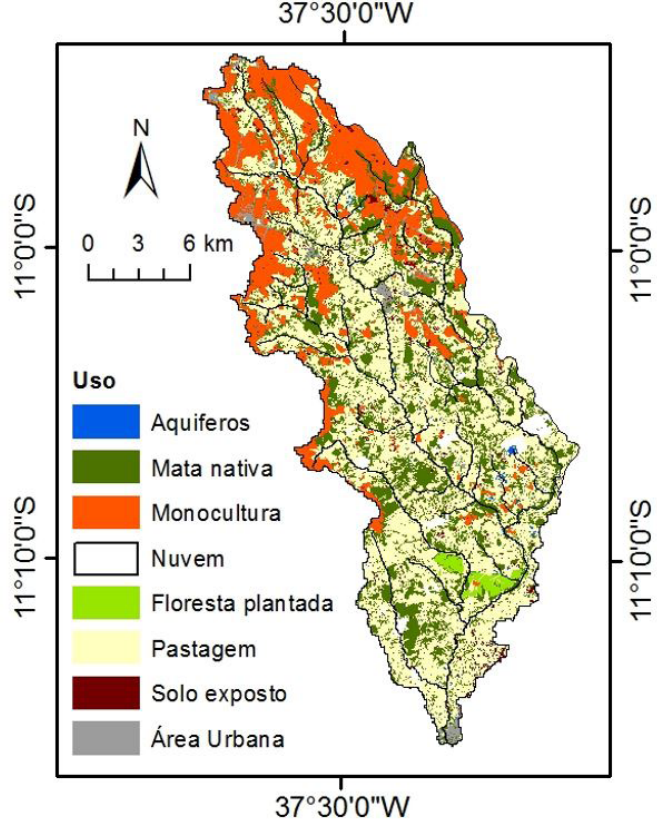

Figura 2. Mapa de uso da terra na Bacia Hidrográfica Rio Piauitinga

Figure 2. Land use map of the river basin Piauitinga.

de a bacia manter qualidade e quantidade de água, sendo essa bacia importante captação de água para a região metropolitana de Aracaju.

Apesar do baixo valor observado (23,79\%), a cobertura florestal na bacia foi superior ao observado em outras regiões brasileiras também inseridas no Bioma Mata Atlântica (Oliveira et al., 2008; Eugênio et al., 2011). 
Segundo Moreira (2008), os remanescentes florestais da Bacia do Rio Piauitinga estão localizados em locais de difícil acesso e nas vertentes de maior declividade, fatores

Tabela 2. Classes de uso e cobertura da terra mapeadas na Bacia do Rio Piauitinga.

Table 2. Land use coverclassesmappedinthe river basin Piauitinga.

\begin{tabular}{lrc}
\multicolumn{1}{c}{ Uso do solo } & Área $\left(\mathbf{k m}^{\mathbf{2}}\right)$ & Área (\%) \\
\hline Aquíferos superficiais & 0,27 & 0,07 \\
\hline Área urbana & 7,71 & 1,87 \\
Monocultura & 77,12 & 18,69 \\
Nuvem & 8,07 & 1,95 \\
Pastagem & 208,10 & 50,43 \\
Solo exposto & 9,16 & 2,22 \\
\hline Floresta plantada & 4,04 & 0,98 \\
Vegetação nativa & 98,19 & 23,79 \\
Total & $\mathbf{4 1 2 , 6 6}$ & $\mathbf{1 0 0}$ \\
\hline
\end{tabular}

que permitiram a manutenção desses remanescentes florestais.

\subsection{Métricas da paisagem}

As métricas da paisagem encontradas na Bacia do Rio Piauitinga podem ser observadas na Tabela 3. A área de classe (CA) e o percentual de fragmentos da classe (PLAND) demonstram que a paisagem da bacia apresenta predomínio de pastagem; os segundos maiores CA e PLAND foram os fragmentos da classe mata nativa, que ocupam área de 9.819,0 ha, que corresponde a $24,8 \%$ da bacia. A área de classe monocultura apresenta alto valor na paisagem e as demais classes apresentam valores baixos (Tabela 3).

A vegetação nativa apresenta, dentre as classes, o maior número de fragmentos (NP), já a pastagem

Tabela 3. Índices de ecologia da paisagem calculados para a Bacia do Rio Piauitinga.

Table 3. Landscape ecology indices calculated for the river basin Piauitinga.

\begin{tabular}{|c|c|c|c|c|c|}
\hline \multirow{2}{*}{ Uso } & \multicolumn{5}{|c|}{ Tamanho e densidade } \\
\hline & CA & PLAND & NP & LPI & AREA_MN \\
\hline Monocultura & 7712,3 & 19,6 & 282,0 & 7,4 & 18,5 \\
\hline Pastagem & 20810,1 & 48,0 & 879,0 & 35,1 & 2,5 \\
\hline Vegetação nativa & 9819,0 & 24,8 & 9323,0 & 1,6 & 0,8 \\
\hline Área urbana & 771,3 & 1,9 & 227,0 & 0,3 & 2,4 \\
\hline Floresta plantada & 404,4 & 1,0 & 174,0 & 0,3 & 33,5 \\
\hline Aquíferos & 26,5 & 0,0 & 56,2 & 0,0 & 0,1 \\
\hline Solo exposto & 916,5 & 2,3 & 1872,0 & 0,0 & 0,4 \\
\hline \multirow{2}{*}{ Uso } & \multicolumn{2}{|c|}{ Borda } & \multicolumn{3}{|c|}{ Área central } \\
\hline & TE & ED & TCA & NDCA & CAI_MN \\
\hline Monocultura & $850.425,0$ & 21,7 & 4208,9 & 369,0 & 19,5 \\
\hline Pastagem & $3664.705,0$ & 93,5 & 8156,0 & 2693,0 & 2,5 \\
\hline Vegetação nativa & $3704.685,0$ & 94,5 & 2371,0 & 1751,0 & 0,8 \\
\hline Área urbana & $352.825,0$ & 9,0 & 0,0 & 0,0 & 0,0 \\
\hline Floresta plantada & 34500,00 & 0,8 & 18,3 & 102,9 & 0,2 \\
\hline Aquíferos & $24.240,0$ & 0,6 & 0,0 & 0,0 & 0,0 \\
\hline Solo exposto & $583.735,0$ & 14,9 & 41,3 & 232,0 & 0,6 \\
\hline \multirow{2}{*}{ Uso } & Forma & \multicolumn{2}{|c|}{ Proximidade } & Dispersão & \\
\hline & SHAPE_MN & PROX_MN & EN_MN & COHESION & \\
\hline Monocultura & 1,6 & 12289,7 & 112,0 & 99,5 & \\
\hline Pastagem & 1,3 & 494596,1 & 24,5 & 100,0 & \\
\hline Vegetação nativa & 1,5 & 775,5 & 29,6 & 98,7 & \\
\hline Área urbana & 1,9 & 1360,5 & 81,3 & 98,9 & \\
\hline Floresta plantada & 2,6 & 1290,0 & 90,3 & 99,0 & \\
\hline Aquíferos & 1,3 & 9,7 & 91,2 & 93,4 & \\
\hline Solo exposto & 1,5 & 13,2 & 86,7 & 94,8 & \\
\hline
\end{tabular}

CA: Área de classe - ha; PLAND: Percentual de fragmentos da classe - \%; NP: Número de fragmentos; LPI: Área do maior fragmento - \%; AREA_MN: Área média dos fragmentos - \%; TE: Total de bordas - ha; ED: Densidade de bordas - m.ha ${ }^{-1}$; TCA: Total da área central - ha; NDCA: Número de áreas centrais disjuntas; CAI_MN: Índice de área central médio - \%; SHAPE_MN: Îndice de forma médio; PROX_MN: Proximidade média entre classes; ENN_MN: Distância média do vizinho mais próximo - m); COHESION: Conectividade - \%. 
tem a maior área de classe e percentual de fragmentos (PLAND), mas apresenta um NP baixo, cerca de 10 vezes menor que o da vegetação nativa. De acordo com Cabacinha et al. (2010), maiores valores de NP indicam maior fragmentação da classe e menores valores indicam união ou extinção de fragmentos de mesma classe. O NP deve ser analisado juntamente com a métrica CA e PLAND: se NP diminui, porém a área de classe e a porcentagem de fragmentos da mesma classe aumentam, isso indica que houve união de fragmentos. No caso da vegetação nativa observa-se que alto NP está associado a uma maior fragmentação dessa classe. A classe solo exposto, apesar de ter baixo $\mathrm{CA}$, apresenta um NP alto em relação ao CA, as classes restantes apresentam baixos valores de NP (Tabela 3 ).

A classe pastagem apresenta o maior percentual da área do maior fragmento (LPI), isso significa que as pastagens na paisagem da Bacia do Rio Piauitinga estão interconectadas, formando uma grande mancha que cobre mais de $30 \%$ da bacia. A classe vegetação nativa obteve um baixo percentual da área do maior fragmento, merecendo atenção a conservação desse fragmento. Souza et al. (2014) observaram LPI de $2,47 \%$ de vegetação nativa, considerado baixo em função de uma matriz antrópica com predomínio de atividades agropastoris, semelhante ao observado neste estudo. O segundo maior LPI foi encontrado na classe monocultura, nesse sentido duas classes da matriz antrópica (pastagem e monocultura) apresentam os maiores fragmentos na paisagem (Tabela 3 ).

As maiores áreas médias dos fragmentos (AREA_MN) foram observadas nas classes monocultura e floresta plantada, mesmo com baixo NP, demonstrando que essas classes apresentam fragmentos extensos e conectados entre si. Baixos valores de AREA_MN foram encontrados para as demais classes, no entanto a vegetação nativa, apesar de ter a segunda maior CA, apresenta uma menor AREA_MN (Tabela 3). Etto et al. (2013) também observaram que os remanescentes florestais da Bacia Hidrográfica do Ribeirão das Pedras apresentam fragmentos com pequena área média, sendo considerados altamente frágeis, tanto que não existe fragmento hábil para a manutenção da biodiversidade genética e animais de porte maior, e os remanescentes de menor extensão, que servem como "trampolins ecológicos" para o fluxo biológico na área, também se encontram intensamente impactados pelas ações antrópicas. Putz et al. (2011), em seu trabalho, observaram que a fragmentação altera a estrutura da floresta, principalmente para os fragmentos florestais menores que 25 ha, os quais são levados para os estados iniciais de sucessão.

Entre os usos antrópicos, a pastagem apresenta a maior densidade de borda (ED) e total de borda (TE). Deve-se destacar também que a monocultura e o solo exposto apresentam alto ED e TE. A vegetação nativa apresenta um forte efeito de borda, considerando que, dentre todos os usos, apresenta as maiores ED e TE, aliadas ao fato de apresentar maior número de fragmentos e baixo valor da área média dos fragmentos, resultando em um grande número de fragmentos de pequeno tamanho com grande efeito de borda (Tabela 3 ). Espécies florestais, em fragmentos isolados, podem ter sua população reduzida, já que estão submetidas aos efeitos do tamanho pequeno do fragmento e da perda de hábitat (Moraes et al., 2015).

A pastagem apresenta o maior valor do total de área central (TCA) e de número de áreas centrais disjuntas (NDCA). A classe monocultura apresenta o maior índice de área central (CAI_MN), o que está de acordo com a maior AREA_MN, em relação às outras classes. A classe mata nativa obteve TCA e NDCA menores que os da classe pastagem. O CAI_MN da mata nativa pode ser considerado muito baixo, quando comparado com as duas principais classes antrópicas, pastagem e monocultura (Tabela 3).

Jesus (2013) observou em uma bacia hidrográfica no Bioma Mata Atlântica, coberta principalmente por monocultura e pastagem no Estado de Sergipe, que os fragmentos menores sofrem maior efeito de borda e redução no índice de área central (CAI_MN). Recoberta predominantemente por monocultura e pastagem, a Bacia do Rio Piauitinga apresenta resultados semelhantes: dentre as classes de uso, a vegetação nativa é a classe que tem uma das menores áreas médias dos fragmentos e, consequentemente, menores áreas centrais (Tabela 3 ).

No índice de forma médio (SHAPE_MN), o quanto mais próximo de 1 , menor o efeito de borda no fragmento. Na paisagem da Bacia do Rio Piauitinga as classes de uso da terra apresentam baixos valores de SHAPE_MN, variando de 1,3 a 1,9, ou seja, os fragmentos das diferentes classes apresentam formas arredondadas, com exceção da floresta plantada. A vegetação nativa apresenta um dos menores SHAPE_MN, expondo menor efeito de borda (Tabela 3). O índice de forma 
próximo a 1 indica que os fragmentos têm forma mais arredondada, que minimiza a relação borda/área (Primack \& Rodrigues, 2001), assim possuem menos área de borda que afeta e, em longo prazo, altera a matriz de vegetação nativa.

A classe pastagem apresenta um maior número de fragmentos com borda, de acordo com a proximidade média entre classes (PROX_MN). A monocultura também obteve um alto PROX_MN, entretanto a mata nativa, apesar de ser a segunda maior área de classe na paisagem, apresenta uma das menores proximidades média (Tabela 3). Essa métrica é fortemente influenciada pela redução de área das classes e pela proximidade (considerando o raio de busca) entre remanescentes de mesma classe na paisagem (Cabacinha et al., 2010).

O ENN_MN na classe monocultura foi a maior distância observada, seguido pelo da classe floresta plantada, aquíferos e solo exposto. A pastagem tem o menor ENN_MN, demonstrando que os fragmentos dessa classe estão muito próximos. Os fragmentos da vegetação nativa têm boa proximidade, apresentando assim um baixo ENN_MN (29,6 m), o que permite uma melhor locomoção da fauna silvestre entre os fragmentos e um maior fluxo de pólen e sementes (Tabela 3). O valor da distância média do vizinho mais próximo pode ser considerado baixo quando comparado aos do trabalho de Souza et al. (2014), que encontraram uma distância média dos fragmentos de 38,9 m, valor considerado baixo.

A classe pastagem tem uma conectividade (COHESION) completa entre seus fragmentos na paisagem da Bacia do Rio Piauitinga. A vegetação nativa, além de apresentar uma boa proximidade, apresenta também alta conectividade, de $98,7 \%$, constituindo assim corredores ecológicos essenciais para deslocamento da fauna silvestre (Tabela 3). De um modo geral, quanto menor a distância entre fragmentos, maior a possibilidade de dispersão das espécies (Caldas \& Francelino, 2009).

As métricas de área central (núcleo) dos fragmentos de vegetação nativa estão apresentadas na Tabela 4, tendo sido geradas por meio da simulação de quatro diferentes distâncias sob efeito de borda, a fim de se verificar se os valores de área central dos fragmentos de Mata Atlântica da Bacia do Rio Piauitinga são afetados pela distância de borda.

Observou-se que conforme aumenta o efeito de borda uma redução no total de área central ocorre, sendo próximo a zero nos fragmentos muito pequenos. Os fragmentos das classes pequena, média e grande, em função de sua maior área, mesmo nas maiores distâncias de borda, mantiveram sua área central (Tabela 4).

Nos fragmentos muito pequenos, o TCA chega a 3,4 ha para uma distância de borda de $90 \mathrm{~m}$, valor muito baixo, próximo de zero. Isso significa que, sob tais condições, essa classe de tamanho encontra-se totalmente dominada pelo efeito de borda, estando todos os fragmentos susceptíveis à influência completa da matriz (Juvanhol et al., 2011). Jesus (2013), utilizando uma distância de borda de $35 \mathrm{~m}$ em uma bacia inserida na Mata Atlântica do Estado de Sergipe, observaram que os fragmentos menores sofreram maior efeito de borda no total de área central.

Os fragmentos pequenos, mesmo com uma distância de borda de $90 \mathrm{~m}$, apresentaram um bom TCA, com 190,7 ha, superior ao TCA de fragmentos maiores,

Tabela 4. Índices de ecologia da paisagem relativos às métricas de área central.

Table 4. Landscape ecology indices for the central area metrics.

\begin{tabular}{|c|c|c|c|c|c|c|}
\hline \multirow{2}{*}{$\begin{array}{c}\text { Distância de } \\
\text { borda }(\mathbf{m})\end{array}$} & \multicolumn{3}{|c|}{ Muito pequeno ( $<5$ ha) } & \multicolumn{3}{|c|}{ Pequeno (5-10 ha) } \\
\hline & TCA & NDCA & CAI_MN & TCA & NDCA & CAI_MN \\
\hline 30 & 166,3 & 301,0 & 15,4 & 1076,7 & 791,0 & 18,6 \\
\hline 60 & 30,2 & 78,0 & 2,7 & 455,0 & 280,0 & 7,3 \\
\hline 90 & 3,4 & 14,0 & 0,2 & 190,7 & 120,0 & 2,7 \\
\hline \multirow{2}{*}{$\begin{array}{c}\text { Distância de } \\
\text { borda }(\mathbf{m})\end{array}$} & \multicolumn{3}{|c|}{ Médio (10-100 ha) } & \multicolumn{3}{|c|}{ Grande (> 100 ha) } \\
\hline & TCA & NDCA & CAI_MN & TCA & NDCA & CAI_MN \\
\hline 30 & 420,6 & 120,0 & 27,9 & 1528,2 & 424,0 & 16,2 \\
\hline 60 & 249,5 & 43,0 & 16,1 & 980,7 & 144,0 & 10,4 \\
\hline 90 & 143,3 & 32,0 & 9,1 & 647,5 & 75,0 & 6,9 \\
\hline
\end{tabular}

TCA: Total da área central; NDCA: Número de áreas centrais disjuntas; CAI_MN: Índice de área central médio. 
como o da classe de tamanho médio (143,3 ha). Os fragmentos grandes, em função da sua maior área. mantêm os totais de área central em todas as distâncias de borda (Tabela 4).

A métrica número de áreas centrais disjuntas (NDCA) reduz-se conforme aumenta a distância da borda nos fragmentos muito pequenos, pequenos, médios e grandes, porém não se aproximando do valor zero (Tabela 4). Juvanhol et al. (2011), avaliando a fragmentação florestal dos parques estaduais do Forno Grande e da Pedra Azul, no Espírito Santo, observaram que o número de áreas centrais disjuntas decresceu com aumento da distância de borda, sendo esse decréscimo menor nos fragmentos maiores.

$\mathrm{O}$ fragmento de tamanho pequeno apresenta maior NDCA desde a menor distância de borda, de $30 \mathrm{~m}$, com NDCA de 791, até uma distancia de $90 \mathrm{~m}$, apresentando um NDCA de 120. Os fragmentos grandes apresentam um efeito de borda menor em relação ao NDCA, porém menor que os fragmentos pequenos (Tabela 4 ).

A classe de tamanho de fragmentos muito pequenos obteve os menores valores de NDCA, variando de 301, para a menor distância, e 14, para a distância de borda de 90 m. Silva et al. (2015) definem que o aumento da distância de borda acarreta em uma redução do NDCA, isso porque o efeito de borda atua nos fragmentos e suas irregularidades, transformando figuras geométricas complexas em superfícies que tendem à circularidade e, assim, ao longo das simulações, obtêm-se valores menores para o NDCA. Também se observa que o total de área central é muito baixo, correspondendo a 3,4 ha para a classe muito pequeno e a uma distância de borda de $90 \mathrm{~m}$, o que reduz o NDCA (Tabela 4).

O índice de área central médio (CAI_MN) apresenta um padrão semelhante às métricas TCA e NDCA, com redução dos valores conforme se aumenta a distância de borda. Os menores fragmentos apresentam maior redução do CAI_MN dentre os demais tamanhos de fragmento. Dessa forma, os fragmentos de menor tamanho são mais susceptíveis à perda de área central, o que compromete a manutenção de processos ecológicos como polinização e dispersão.

$\mathrm{O}$ índice de área central médio dos fragmentos muito pequenos foi de 15,4\% para a menor distância de borda, de $30 \mathrm{~m}$, e aproxima-se de zero para distância de borda de $90 \mathrm{~m}$, estando praticamente toda a sua área central sob efeito de borda. É necessário aumentar a conectividade dos fragmentos muito pequenos, que podem fazer o papel de trampolim ecológico para a fauna silvestre. Os fragmentos de tamanho médio apresentaram os maiores valores de CAI_MN, desde a distância de borda de $30 \mathrm{~m}$ até a de $90 \mathrm{~m}$, variando de $27,9 \%$ a $9,1 \%$.

\section{CONCLUSÕES}

A Bacia do Rio Piauitinga apresenta uma paisagem cuja matriz está representada por pastagens, que formam a área de maior fragmento e a maior área central. A parte superior da bacia encontra-se recoberta por monoculturas agrícolas, sendo recomendado o reflorestamento nessa região, pois é onde estão as principais nascentes da bacia.

A vegetação nativa é a segunda maior classe de cobertura, porém está fragmentada em numerosos remanescentes de tamanho pequeno e com grande efeito de borda. Independentemente da classe de tamanho, conforme se aumenta a distância de borda reduz-se a área central, no que os fragmentos da classe muito pequenos foram os mais afetados, sendo indicado o estabelecimento de corredores ecológicos.

\section{STATUS DA SUBMISSÃO}

Recebido: 13 maio, 2016

Aceito: 3 jul., 2016

\section{AUTOR(ES) PARA CORRESPONDÊNCIA}

\section{André Almeida}

Departamento de Engenharia Agrícola DEAGRI, Universidade Federal de Sergipe UFS, Av. Marechal Rondon, s/n, Jd. Rosa Elze, CEP 49100-000, São Cristóvão, SE, Brasil e-mail: andreqa@gmail.com

\section{REFERÊNCIAS}

Banks-Leite C, Ewers RM, Kapos V, Martensen AC, Metzger JP. Comparing species and measures of landscape structure as indicators of conservation importance. Journal of Applied Ecology 2011; 48(3): 706-714. http://dx.doi. org/10.1111/j.1365-2664.2011.01966.x. 
Cabacinha CD, Castro SS, Gonçalves DA. Análise da estrutura da paisagem da alta bacia do Rio Araguaia na savana brasileira. Revista Floresta 2010; 40(4): 675-690.

Caldas AJS, Francelino MR. Fragmentação florestal na Serra da Concórdia, Vale do Paraíba: caracterização como subsídio a preservação da Mata Atlântica. Floresta e Ambiente 2009; 16(2): 10-19.

Etto TL, Longo RM, Arruda DR, Invenioni R. Ecologia da paisagem de remanescentes florestais na Bacia Hidrográfica do Ribeirão das Pedras - Campinas-SP. Revista Árvore 2013; 37(6): 1063-1071. http://dx.doi.org/10.1590/S010067622013000600008

Eugênio FC, Santos AR, Louzada FLRO, Pimentel LB, Moulin JV. Identificação de áreas de preservação permanente no município de Alegre utilizando geotecnologia. Cerne 2011; 17(4): 563-571. http://dx.doi.org/10.1590/S010477602011000400016 .

Ferreira RA, Aguiar Netto AO, Santos TIS, Santos BL, Matos EL. Nascentes da sub-bacia hidrográfica do rio Poxim, estado de Sergipe: da degradação à restauração. Revista Árvore 2011; 35(20): 265-277. http://dx.doi. org/10.1590/S0100-67622011000200011.

Jesus EN. Avaliação dos fragmentos florestais da Bacia Hidrográfica do Rio Poxim (Sergipe - Brasil) para fins restauração ecológica [dissertação]. São Cristóvão: Universidade Federal de Sergipe; 2013.

Juvanhol RS, Fiedler NC, Santos AR, Pirovani DB, Louzada FLRO, Dias HM et al. Análise espacial de fragmentos florestais: caso dos Parques Estaduais de Forno Grande e Pedra Azul, estado do Espírito Santo. Revista Floresta e Ambiente 2011; 18(4): 353-364. http://dx.doi.org/10.4322/ floram.2011.055

Magalhães LTS, Gomes JBV, Vasco NA, Aguiar Netto AO, Ferreira RA. Caracterização geo-pedológica das áreas de nascentes na bacia hidrográfica do rio Piauitinga, Sergipe, Brasil. Revista Ambi-Agua 2012; 7(1): 169-181. http:// dx.doi.org/10.4136/ambi-agua.767.

McGarigal K. Fragstats: Fragstats help - versão 4.2. Computer software program produced by the author at the University of Massachusetts. Massachusetts: Amherst; 2013. 182 p.

Metzger JP. Conservation issues in the Brazilian Atlantic Forest. Biological Conservation 2009; 142(6): 1138-1140. http://dx.doi.org/10.1016/j.biocon.2008.10.012.
Moraes MCP, Mello K, Toppa RH. Análise da paisagem de uma zona de amortecimento como subsídio para o planejamento e gestão de unidades de conservação. Revista Árvore 2015; 39(2): 1-8. http://dx.doi.org/10.1590/010067622015000100001 .

Moreira FD. Geotecnologia aplicada à sub-bacia hidrográfica do rio Piauitinga e suas relações ambientais [dissertação]. São Cristóvão: Universidade Federal de Sergipe; 2008.

Nascimento HR, Abreu YV. Geração de informações sobre a agricultura de energia por meio das geotecnologias. Interações 2012; 13(2): 181-189.

Oliveira JT, Moreau AMSS, Paiva AQ, Menezes AA, Costa OV. Características físicas e carbono orgânico de solos sob diferentes tipos de uso da terra. Revista Brasileira de Ciência do Solo 2008; 32(1): 2821-2829. http://dx.doi. org/10.1590/S0100-06832008000700028.

Planet Labs Inc. San Francisco; 2016. [cited 2016 May 13]. Disponível em: http://blackbridge.com/rapideye/upload/ RE_Product_Specifications_ENG.pdf

Primack RB, Rodrigues E. Biologia da conservação. 3. ed. Londrina: Editora Vida; 2001.

Putz S, Groeneveld J, Alves LF, Metzger JP, Huth A. Fragmentation drives tropical forest fragments to early successional states: A modelling study for Brazilian Atlantic forests. Ecological Modelling 2011; 222(12): 1986-1997. http://dx.doi.org/10.1016/j.ecolmodel.2011.03.038.

Ribeiro MC, Metzger JP, Martensen AC, Ponzoni FJ, Hirota MM. The Brazilian Atlantic Forest: how much is left, and how is the remaining forest distribuited? implications for conservation. Biological Conservation 2009; 142(6): 1141 1153. http://dx.doi.org/10.1016/j.biocon.2009.02.021.

Silva MSF, Souza RM. Spatial patterns of forest fragmentation in the Flona Ibura - Sergipe. Mercator 2014; 13(3): 121-137. http://dx.doi.org/10.4215/RM2014.1303.0009.

Silva RA, Pereira JAA, Barros DA, Borges LAC, Teixeira MD, Acerb IFW. avaliação da cobertura florestal na paisagem de Mata Atlântica no ano de 2010, na região de Ouro Preto - MG. Cerne 2015; 21(2): 301-309. http:// dx.doi.org/10.1590/01047760201521021539.

Souza GC, Zanella L, Borém RAT, Carvalho LMT, Alves HMR, Volpato MML. Análise da fragmentação florestal da Área de Proteção Ambiental Coqueiral, Coqueiral MG. Ciência Florestal 2014; 24(3): 631-644. http://dx.doi. org/10.5902/1980509815743. 